\title{
An investigation of 10 Y-STR loci and the detection of specific haplotype frequencies in Turkish population
}

\author{
Aydın Rüstemoglu ${ }^{1}$, Güvem Gümüs Akay², Halil Gürhan Karabulut ${ }^{3}$, Ahmet Kadıkıran ${ }^{4}$, \\ Işık Bökesoy $^{3}$
}

\footnotetext{
${ }^{1}$ Gaziosmanpaşa University, Faculty of Medicine, Department of Medical Biology, Tokat, Turkey;

${ }^{2}$ Ankara University, Brain Studies Application and Research Center, Ankara, Turkey;

${ }^{3}$ Ankara University, Faculty of Medicine, Department of Medical Genetics, Ankara, Turkey;

${ }^{4}$ Ankara University, Faculty of Science, Department of Molecular Biology, Ankara, Turkey.

E-mail: arustamov@gop.edu.tr
}

Received 26 May 2011; revised 16 June 2011; accepted 30 June 2011.

\section{ABSTRACT}

This study is to survey 10 Y-STR loci in 241 males from Turkey.

In this study, the 241 healthy and unrelated males living in different parts of Turkey for at least three generations were included. Genomic DNAs were isolated from peripheral blood samples by standard phenol-chloroform extraction method. 10 Y-STR loci including DYS19, DYS385a/b, DYS388, DYS389I/II, DYS390, DYS391, DYS392, DYS393, and YCAIIa/b were analyzed by using PCR and denaturing PAGE.

Allele frequencies, gene diversities and haplotype frequencies were analyzed. Gene diversity per locus varied from 0.5788 (DYS388) to 0.8903 (DYS385a/b). The numbers of haplotypes in minHt recommended by YCC and Ht10 have been 208 and 186, respectively. When our minHt haplotypes frequencies compared with the other seven populations, we have found statistically significant differences between our results and other populations $(p<0.01)$ except that Czech population $(p>0.05)$. We suggest that an alternative haplotype designated as $a \mathrm{Ht}$ maybe alternative to minHt in respect of its Y-STR content with the highest gene diversity value. The aHt haplotype has found a higher discriminatory potential than minHt haplotype with a better $\boldsymbol{P}_{\boldsymbol{d} \text { combined }}$ value (0.9999936 vs $0.9999836)$ and has higher average gene diversity per locus (0.7834 vs 0.7518$)$ in Turkish population.

aHt haplotype can be proposed as an alternative to minHt in paternity testing and forensic medicine applications involving Turkish male population. This study has also provided additional information to the framework of variation involving 10 Y-STR loci as well as a further contribution to the Y-STR database for Turkish male population.

Keywords: Y Chromosome; Y-STR; Polymorphism; Haplotype; Turkey

\section{INTRODUCTION}

Human Y chromosome has been known to display comparatively low levels of polymorphisms in contrast with autosomal chromosomes. Nonetheless, there are many types of Y-chromosome specific polymorphisms identified in the non-recombining region of Y (NRY) including RFLPs, Y Alu and microsatellite polymorphisms [1, 2]. Microsatellites or short tandem repeats (STRs) are dispersed throughout the genome and are known to be highly polymorphic; hence, each microsatellite locus generally has many alleles due to variable number of repeat units $[3,4]$.

Allelic genotyping of STRs does not require the use of complex molecular techniques, since amplifications and visualization of PCR products make it easy. Y-chromosome specific STRs (Y-STRs) are chosen as more informative in paternity testing, forensic applications and the study of population histories due to the haploid state of Y chromosome which ensures both the transmittance by the paternal lineages and the lack of recombination in NRY, excluding pseudoautosomal regions (PARs) [5$11]$.

Allelic and haplotypic distributions of Y-STRs have shown significant differences in different geographical regions, ethnical groups and communities [12-18]. Therefore, allelic and haplotypic frequencies of Y-STRs should be determined in a male population prior to any interpretations of forensic analysis and paternity testing [6,8-11,19]. In this study, allelic and haplotypic frequent- 
cies involving 10 Y-STR loci: 8 Y-STR loci as recommended by Y Chromosome Consortium (YCC) plus DYS388 and YCAIIa/b-have been determined with such a necessity in a representative group of Turkish population in order to make comparisons with other populations.

\section{MATERIALS AND METHODS}

10 Y-STR loci were analyzed which included eight loci recommended by YCC for minimal haplotype $(\operatorname{minH} t)$ DYS19, DYS385a/b, DYS389I/II, DYS390, DYS391, DYS392, and DYS393 $[20,21]$ and the additional two: DYS388 and YCAIIa/b.

Healthy and unrelated 241 males living in different parts of Turkey for at least three generations were included in this study. The written informed consents were obtained from the study subjects and the study protocol was approved by the ethics committee of Ankara University Medical Faculty.

Genomic DNAs were isolated from peripheral blood samples by standard phenol-chloroform extraction method [22]. Four multiplex PCR analyses were carried out with locus specific primers in a total volume of $25 \mu \mathrm{l}$ reaction mixture, containing; 50 - 150 ng genomic DNA, $10 \mathrm{mM}$ Tris- $\mathrm{HCl}, 50 \mathrm{mM} \mathrm{KCl}, 1.5 \mathrm{mM} \mathrm{MgCl}_{2}, 0.1-0.8$ $\mu \mathrm{M}$ of each primer, $200 \mu \mathrm{M}$ of each dNTP (Sigma), 5 $\mu \mathrm{g}$ BSA and 1 unit Taq DNA polymerase (Invitrogen) [23]. Y-STRs amplified in combination in multiplex PCR are as follows: DYS389I, DYS389 II and DYS390 in multiplex I; DYS392 and DYS393 in multiplex II; DYS19 and DYS388 in multiplex III; DYS385a/b and DYS391 in multiplex IV; while YCAIIa/b loci were amplified separately. Cycling conditions were as follows: 32 cycles of $94^{\circ} \mathrm{C}-1 \mathrm{~min}, 54^{\circ} \mathrm{C}-1 \mathrm{~min}, 72^{\circ} \mathrm{C}-1 \mathrm{~min}$ for multiplex I, II, IV and YCAIIa/b; 35 cycles of $94^{\circ} \mathrm{C}$ $1 \mathrm{~min}, 55^{\circ} \mathrm{C}-1 \mathrm{~min}, 72^{\circ} \mathrm{C}-60 \mathrm{~min}$ for multiplex III. An initial denaturation at $94^{\circ} \mathrm{C}-2.5 \mathrm{~min}$ and a final extension at $72^{\circ} \mathrm{C}-10 \mathrm{~min}$ were performed before and after each cycling reactions. Female DNA was used as a negative control in every run.

Amplified products were separated by $6 \%$ denaturing polyacrylamide gel electrophoresis (PAGE) for $3 \mathrm{~h}$ at $1500 \mathrm{~V}$. Visualization of PCR products was carried out by a modification of the silver staining method of Santos, et al. [23]. Gels were fixed for $15 \mathrm{~min}$ at room temperature in $10 \%(\mathrm{v} / \mathrm{v})$ ethanol which were treated with $1 \%$ $(\mathrm{v} / \mathrm{v})$ nitric acid for 3 min with agitation thereafter. Gels were then rinsed in deionized water for $1 \mathrm{~min}$ and treated with $0.2 \%(\mathrm{w} / \mathrm{v})$ silver nitrate and $0.1 \%(\mathrm{v} / \mathrm{v})$ formaldehyde solution for $25 \mathrm{~min}$ with agitation. Gels were rinsed for a few seconds in deionized water, and developed in an aqueous solution of $3 \% \mathrm{Na}_{2} \mathrm{CO}_{3}(\mathrm{w} / \mathrm{v}), 100 \mu \mathrm{l} / \mathrm{L} 2 \%$ (w/v) $\mathrm{Na}_{2} \mathrm{~S}_{2} \mathrm{O}_{3}$ and $0.1 \%$ formaldehyde until the bands were well visualized. Staining was ended with a fixative solution.

Allelic genotyping were carried out by using the defined DNA size markers (Fermentase, pUC Mix Marker, 8; ФX174 RF DNA/BsuRI [HaeIII] Marker, 9) and selfmade ladders as standards.

Allele frequencies, gene diversities $(\mathrm{H})$, haplotype frequencies and genetic differentiation between populations were computed using Arlequin 3.1.1 software. Allele frequencies were calculated by gene counting, while $H$ was computed for each locus according to the by formula (1), where $n$ is the number of samples, $k$ is the number of haplotypes, and $p_{i}$ is the frequency of $i$-th haplotype [24].

$$
H=\frac{n}{n-1}\left(1-\sum_{1}^{k} p_{i}^{2}\right)
$$

The Combined Power of Discrimination $\left(P_{d \text { combined }}\right)$ for haplotypes was calculated using the by formula (2), where $P_{d i}$ is Power of Discrimination of $i$-th locus [25].

$$
P_{d \text { combined }}=1-\pi_{i=1}^{n}\left(1-P_{d i}\right)
$$

\section{RESULTS AND DISCUSSION}

10 Y-STRs have been analyzed for diversity in 241 healthy and unrelated male individuals from Turkey. Observed allele or genotype frequencies of the 10 Y-STR loci have been given in Table 1. Variations in the number of individuals for certain loci have been brought about by some technical problems not anticipated.

Gene diversity values for each 10 Y-STR loci have been given in Table 1 . The lowest gene diversity $(0.5788)$ has been found in DYS388 locus, wherein the most frequent allele has been allele 13 with a frequency of $62.08 \%$. This result has been in accord with the data reported by YCC [21]. The highest gene diversity $(0.8903)$ has been found in DYS385 locus, wherein the most frequent allele has been allele 14 with a frequency of $17.62 \%$ (Table 1 )

The observed number of haplotypes and their frequencies involving minHt and Ht10 haplotypes in this current survey have been tabulated in Table 2. The number of haplotypes detected for minHt is 208 and 186 for $H t 10$. Each haplotype belonging to $H t 10$ have been found to be unique while the same holds for $\mathrm{minHt}$ with the exception of H15 which has been detected in two individuals. Gene diversity, average gene diversity per locus and Combined Power of Discrimination $\left(P_{d \text { combined }}\right)$ values for $H t 10$ and minHt haplotypes have been given in Table 3.

minHt haplotypes detected in this study group have been compared with seven other populations: Croatian 
Table 1. Detected allele frequencies and gene diversities of the Y-STR loci in Turkish population.

\begin{tabular}{|c|c|c|c|c|c|c|c|c|c|c|}
\hline Allele & $\begin{array}{c}\text { DYS19 } \\
(n=228)\end{array}$ & $\begin{array}{l}\text { DYS385 }^{*} \\
(n=454)\end{array}$ & $\begin{array}{l}\text { DYS388 } \\
(n=234)\end{array}$ & $\begin{array}{l}\text { DYS389I } \\
(n=239)\end{array}$ & $\begin{array}{c}\text { DYS389II } \\
(n=234)\end{array}$ & $\begin{array}{l}\text { DYS390 } \\
(n=241)\end{array}$ & $\begin{array}{l}\text { DYS391 } \\
(n=224)\end{array}$ & $\begin{array}{l}\text { DYS392 } \\
(n=237)\end{array}$ & $\begin{array}{l}\text { DYS393 } \\
(n=241)\end{array}$ & $\begin{array}{c}\text { YCAII }^{*} \\
(n=392)\end{array}$ \\
\hline 7 & & 0.0022 & & & & & & & & \\
\hline 8 & & 0.0176 & & & & & & 0.0042 & & \\
\hline 9 & & 0.0330 & & & & & 0.0130 & 0.0042 & 0.0124 & \\
\hline 10 & & 0.0969 & & & & & 0.1732 & 0.0633 & 0.0166 & \\
\hline 11 & & 0.0903 & 0.0083 & 0.0167 & & & 0.5541 & 0.4262 & 0.0747 & \\
\hline 12 & & 0.1189 & 0.0083 & 0.0628 & & & 0.2338 & 0.2321 & 0.3942 & \\
\hline 13 & 0.0342 & 0.1608 & 0.6208 & 0.2929 & & & 0.0260 & 0.1941 & 0.3361 & \\
\hline 14 & 0.2222 & 0.1762 & 0.0458 & 0.4059 & & & & 0.0422 & 0.1162 & 0.0025 \\
\hline 15 & 0.4145 & 0.1057 & 0.0958 & 0.1590 & & & & 0.0295 & 0.0498 & 0.0 \\
\hline 16 & 0.2863 & 0.0815 & 0.1542 & 0.0628 & & & & 0.0042 & & 0.0 \\
\hline 16.3 & & 0.0066 & & & & & & & & \\
\hline 17 & 0.0385 & 0.0441 & 0.0542 & & & & & & & 0.0228 \\
\hline 17.2 & & 0.0022 & & & & & & & & \\
\hline 17.3 & & 0.0396 & & & & & & & & \\
\hline 18 & 0.0043 & 0.0022 & 0.0083 & & & & & & & 0.0533 \\
\hline 20 & & 0.0066 & & & & 0.0041 & & & & 0.1675 \\
\hline 21 & & 0.0022 & & & & 0.0083 & & & & 0.1066 \\
\hline 22 & & & & & & 0.0581 & & & & 0.1878 \\
\hline 23 & & & & & & 0.2116 & & & & 0.1193 \\
\hline 24 & & & & & & 0.3361 & & & & 0.0203 \\
\hline 25 & & & & & & 0.2739 & & & & 0.0 \\
\hline 26 & & & & & 0.0085 & 0.0913 & & & & 0.0025 \\
\hline 27 & & & & & 0.0427 & 0.0166 & & & & \\
\hline 28 & & & & & 0.1667 & & & & & \\
\hline 29 & & & & & 0.1838 & & & & & \\
\hline 30 & & & & & 0.2564 & & & & & \\
\hline 31 & & & & & 0.1453 & & & & & \\
\hline 32 & & & & & 0.1410 & & & & & \\
\hline 33 & & & & & 0.0556 & & & & & \\
\hline $\mathbf{H}$ & 0.6971 & 0.8903 & 0.5788 & 0.7191 & 0.8293 & 0.7583 & 0.6101 & 0.7232 & 0.7126 & 0.8087 \\
\hline
\end{tabular}

*Allele frequencies was calculated including two genomic copies.

Table 2. Detected number of haplotypes and their frequencies in minHt and Ht10 haplotypes surveyed in Turkish population in this study.

\begin{tabular}{|c|c|c|c|c|c|c|c|c|c|c|c|c|}
\hline \multirow{3}{*}{ Haplotypes } & \multicolumn{12}{|c|}{ Ht10* } \\
\hline & \multicolumn{9}{|c|}{$\operatorname{minH} t^{* *}$} & \multirow[b]{2}{*}{ YCAIIa/b } & \multirow[b]{2}{*}{ DYS388 } & \multirow[b]{2}{*}{$N^{* * *}$} \\
\hline & DYS19 & DYS385a/b & DYS389I & DYS389II & DYS390 & DYS391 & DYS392 & DYS393 & $N^{* *}$ & & & \\
\hline HPT1 & 15 & 11,11 & 13 & 29 & 23 & 10 & 11 & 12 & 1 & $24 / 24$ & 16 & 1 \\
\hline HPT3 & 15 & 13,9 & 13 & 29 & 25 & 11 & 13 & 12 & 1 & $20 / 20$ & 15 & 1 \\
\hline HPT4 & 15 & 13,12 & 13 & 29 & 24 & 12 & 11 & 13 & 1 & $23 / 20$ & 13 & 1 \\
\hline HPT5 & 15 & 13,9 & 13 & 29 & 25 & 12 & 13 & 12 & 1 & $23 / 20$ & 13 & 1 \\
\hline HPT6 & 15 & 15,14 & 13 & 30 & 24 & 10 & 11 & 13 & 1 & $23 / 20$ & 12 & 1 \\
\hline HPT7 & 15 & 15,10 & 13 & 29 & 24 & 12 & 13 & 12 & 1 & $24 / 20$ & 13 & 1 \\
\hline HPT8 & 14 & 13,9 & 14 & 32 & 23 & 11 & 12 & 12 & 1 & $22 / 20$ & 16 & 1 \\
\hline HPT9 & 15 & 12,8 & 12 & 29 & 24 & 11 & 14 & 12 & 1 & $21 / 19$ & 15 & 1 \\
\hline HPT10 & 15 & 12,10 & 14 & 32 & 25 & 11 & 11 & 12 & 1 & $22 / 19$ & 16 & 1 \\
\hline HPT11 & 15 & 14,12 & 15 & 33 & 24 & 11 & 11 & 12 & 1 & $22 / 17$ & 16 & 1 \\
\hline HPT12 & 16 & 15,12 & 15 & 33 & 24 & 11 & 11 & 12 & 1 & $22 / 22$ & 16 & 1 \\
\hline HPT13 & 15 & $17.3,11$ & 13 & 28 & 24 & 10 & 12 & 13 & 1 & $22 / 19$ & 13 & 1 \\
\hline HPT14 & 15 & 17,17 & 14 & 31 & 25 & 11 & 11 & 13 & 1 & $20 / 19$ & 13 & 1 \\
\hline HPT16 & 16 & $17.3,11$ & 15 & 31 & 24 & 11 & 11 & 12 & 1 & $22 / 19$ & 17 & 1 \\
\hline HPT17 & 14 & $17.3,14$ & 13 & 31 & 23 & 11 & 11 & 13 & 1 & $22 / 19$ & 13 & 1 \\
\hline HPT18 & 16 & 13,12 & 13 & 31 & 23 & 12 & 11 & 13 & 1 & $21 / 19$ & 16 & 1 \\
\hline HPT19 & 16 & 13,9 & 15 & 33 & 25 & 11 & 11 & 12 & 1 & $22 / 21$ & 17 & 1 \\
\hline HPT20 & 15 & 14,11 & 14 & 31 & 24 & 11 & 11 & 12 & 1 & $22 / 20$ & 13 & 1 \\
\hline HPT21 & 15 & 12,12 & 14 & 31 & 25 & 11 & 12 & 13 & 1 & $23 / 19$ & 13 & 1 \\
\hline HPT22 & 14 & 13,11 & 14 & 30 & 24 & 12 & 11 & 12 & 1 & $22 / 19$ & 16 & 1 \\
\hline HРТ 23 & 16 & 16,11 & 14 & 30 & 26 & 12 & 11 & 12 & 1 & $23 / 19$ & 13 & 1 \\
\hline HРТ 24 & 13 & $17.3,12$ & 14 & 32 & 24 & 12 & 12 & 13 & 1 & $22 / 19$ & 17 & 1 \\
\hline HPT25 & 14 & 16,15 & 14 & 31 & 24 & 11 & 12 & 13 & 1 & $20 / 20$ & 13 & 1 \\
\hline
\end{tabular}




\begin{tabular}{|c|c|c|c|c|c|c|c|c|c|c|c|c|}
\hline HPT26 & 14 & $17.3,13$ & 13 & 30 & 25 & 11 & 13 & 13 & 1 & $20 / 19$ & 13 & 1 \\
\hline HPT27 & 15 & $17.3,15$ & 14 & 31 & 23 & 11 & 11 & 12 & 1 & $22 / 19$ & 16 & 1 \\
\hline HPT28 & 15 & 14,10 & 14 & 30 & 25 & 12 & 13 & 12 & 1 & $23 / 19$ & 13 & 1 \\
\hline HРT29 & 14 & 14,10 & 13 & 30 & 25 & 11 & 11 & 13 & 1 & $23 / 19$ & 16 & 1 \\
\hline HPT30 & 15 & 16,14 & 14 & 30 & 24 & 11 & 13 & 13 & 1 & $23 / 23$ & 13 & 1 \\
\hline HPT31 & 15 & 15,13 & 14 & 30 & 26 & 11 & 11 & 12 & 1 & $22 / 19$ & 13 & 1 \\
\hline HPT32 & 16 & 14,10 & 14 & 32 & 25 & 10 & 11 & 13 & 1 & $23 / 19$ & 13 & 1 \\
\hline HPT33 & 15 & 21,12 & 14 & 33 & 24 & 12 & 11 & 12 & 1 & $22 / 19$ & 15 & 1 \\
\hline HPT34 & 15 & 14,10 & 15 & 30 & 25 & 12 & 11 & 13 & 1 & $22 / 19$ & 13 & 1 \\
\hline HPT35 & 15 & $17.3,13$ & 14 & 30 & 24 & 11 & 12 & 11 & 1 & $22 / 18$ & 16 & 1 \\
\hline HPT36 & 14 & 16,12 & 13 & 30 & 26 & 11 & 11 & 12 & 1 & $23 / 19$ & 13 & 1 \\
\hline HРT37 & 15 & 11,9 & 14 & 30 & 26 & 11 & 8 & 13 & 1 & $22 / 19$ & 15 & 1 \\
\hline HPT38 & 16 & 14,14 & 12 & 29 & 23 & 11 & 10 & 14 & 1 & $22 / 19$ & 13 & 1 \\
\hline HРT39 & 15 & $17.2,15$ & 13 & 30 & 25 & 11 & 10 & 14 & 1 & $22 / 18$ & 16 & 1 \\
\hline HPT40 & 16 & 14,14 & 13 & 30 & 23 & 11 & 10 & 13 & 1 & $23 / 19$ & 13 & 1 \\
\hline HPT41 & 15 & 14,10 & 14 & 30 & 24 & 12 & 11 & 13 & 1 & $22 / 19$ & 15 & 1 \\
\hline HPT42 & 15 & 20,9 & 13 & 30 & 24 & 12 & 10 & 12 & 1 & $22 / 19$ & 13 & 1 \\
\hline HPT43 & 15 & 19,17 & 13 & 29 & 25 & 11 & 10 & 13 & 1 & $22 / 18$ & 16 & 1 \\
\hline HPT44 & 15 & 15,10 & 14 & 31 & 25 & 12 & 11 & 12 & 1 & $23 / 19$ & 13 & 1 \\
\hline HPT45 & 15 & 16,12 & 14 & 31 & 24 & 12 & 10 & 12 & 1 & $22 / 19$ & 15 & 1 \\
\hline HPT46 & 15 & 9,9 & 14 & 31 & 25 & 12 & 11 & 13 & 1 & $22 / 19$ & 13 & 1 \\
\hline HPT47 & 15 & 16,12 & 14 & 33 & 23 & 10 & 11 & 12 & 1 & $22 / 18$ & 16 & 1 \\
\hline HPT48 & 16 & 11,10 & 14 & 32 & 24 & 11 & 10 & 12 & 1 & $23 / 19$ & 13 & 1 \\
\hline HPT49 & 15 & 13,11 & 13 & 30 & 24 & 11 & 12 & 13 & 1 & $22 / 19$ & 15 & 1 \\
\hline HPT50 & 13 & 14,12 & 13 & 32 & 22 & 11 & 11 & 15 & 1 & $22 / 19$ & 13 & 1 \\
\hline HPT51 & 14 & 15,12 & 15 & 33 & 24 & 11 & 12 & 12 & 1 & $22 / 18$ & 16 & 1 \\
\hline HPT52 & 14 & 17,11 & 12 & 29 & 24 & 10 & 11 & 12 & 1 & $23 / 19$ & 13 & 1 \\
\hline HPT53 & 14 & 15,14 & 12 & 30 & 23 & 10 & 11 & 13 & 1 & $22 / 19$ & 15 & 1 \\
\hline HPT54 & 14 & 17,13 & 14 & 32 & 24 & 10 & 11 & 12 & 1 & $22 / 19$ & 13 & 1 \\
\hline HPT55 & 14 & 17,14 & 14 & 32 & 25 & 10 & 13 & 13 & 1 & $22 / 18$ & 16 & 1 \\
\hline HPT56 & 14 & 17,12 & 14 & 32 & 23 & 10 & 12 & 12 & 1 & $23 / 19$ & 13 & 1 \\
\hline HPT57 & 16 & 12,9 & 13 & 31 & 26 & 11 & 11 & 13 & 1 & $22 / 19$ & 15 & 1 \\
\hline HPT58 & 14 & 10,10 & 14 & 32 & 24 & 11 & 11 & 12 & 1 & $22 / 19$ & 13 & 1 \\
\hline HPT59 & 14 & 14,11 & 13 & 31 & 26 & 10 & 13 & 12 & 1 & $22 / 18$ & 16 & 1 \\
\hline HPT60 & 15 & 17,13 & 14 & 32 & 24 & 10 & 12 & 11 & 1 & $23 / 19$ & 13 & 1 \\
\hline HPT61 & 16 & 14,14 & 14 & 31 & 24 & 10 & 12 & 13 & 1 & $22 / 19$ & 15 & 1 \\
\hline HPT62 & 14 & 13,11 & 15 & 32 & 25 & 11 & 13 & 12 & 1 & $22 / 19$ & 13 & 1 \\
\hline HPT63 & 15 & 15,12 & 13 & 31 & 22 & 12 & 11 & 15 & 1 & $22 / 18$ & 16 & 1 \\
\hline HPT64 & 15 & 19,12 & 14 & 32 & 26 & 12 & 13 & 13 & 1 & $23 / 19$ & 13 & 1 \\
\hline HPT65 & 16 & 13,11 & 14 & 29 & 25 & 11 & 10 & 14 & 1 & $22 / 19$ & 15 & 1 \\
\hline HPT66 & 14 & 14,12 & 14 & 31 & 25 & 11 & 11 & 12 & 1 & $22 / 19$ & 13 & 1 \\
\hline HPT67 & 15 & 14,12 & 14 & 33 & 25 & 11 & 12 & 14 & 1 & $22 / 18$ & 16 & 1 \\
\hline HPT68 & 14 & 13,10 & 14 & 31 & 26 & 12 & 13 & 12 & 1 & $23 / 19$ & 13 & 1 \\
\hline HРT69 & 13 & 15,15 & 14 & 32 & 25 & 12 & 11 & 13 & 1 & $21 / 19$ & 13 & 1 \\
\hline HPT70 & 14 & 16,13 & 14 & 32 & 24 & 11 & 12 & 12 & 1 & $22 / 17$ & 16 & 1 \\
\hline HPT71 & 14 & 19,13 & 14 & 33 & 24 & 11 & 12 & 11 & 1 & $22 / 19$ & 19 & 1 \\
\hline HPT72 & 16 & 14,10 & 15 & 33 & 25 & 12 & 12 & 13 & 1 & $23 / 19$ & 13 & 1 \\
\hline HPT73 & 15 & 16,13 & 13 & 30 & 25 & 10 & 10 & 12 & 1 & $20 / 19$ & 16 & 1 \\
\hline HPT74 & 14 & 13,12 & 15 & 29 & 26 & 9 & 11 & 12 & 1 & $22 / 20$ & 16 & 1 \\
\hline HPT75 & 14 & 14,12 & 15 & 28 & 24 & 10 & 12 & 12 & 1 & $22 / 19$ & 16 & 1 \\
\hline HPT76 & 16 & 14,13 & 16 & 29 & 24 & 11 & 12 & 14 & 1 & $20 / 19$ & 13 & 1 \\
\hline HPT77 & 15 & 14,10 & 13 & 27 & 22 & 10 & 13 & 15 & 1 & $20 / 19$ & 13 & 1 \\
\hline HPT78 & 15 & 15,12 & 14 & 28 & 24 & 10 & 13 & 11 & 1 & $22 / 19$ & 16 & 1 \\
\hline HPT79 & 14 & 13,10 & 15 & 29 & 23 & 12 & 13 & 11 & 1 & $21 / 19$ & 17 & 1 \\
\hline HPT80 & 13 & 14,12 & 14 & 30 & 25 & 10 & 12 & 12 & 1 & $22 / 20$ & 13 & 1 \\
\hline HPT81 & 15 & 14,7 & 15 & 28 & 23 & 10 & 13 & 10 & 1 & $21 / 20$ & 13 & 1 \\
\hline HPT82 & 15 & 11,10 & 14 & 27 & 23 & 10 & 11 & 14 & 1 & $20 / 19$ & 13 & 1 \\
\hline HPT83 & 13 & 15,13 & 15 & 28 & 25 & 10 & 11 & 11 & 1 & $20 / 19$ & 13 & 1 \\
\hline HPT84 & 14 & 12,12 & 15 & 29 & 25 & 12 & 12 & 9 & 1 & $24 / 19$ & 13 & 1 \\
\hline HPT85 & 14 & 15,14 & 16 & 29 & 24 & 11 & 11 & 13 & 1 & $21 / 19$ & 13 & 1 \\
\hline HPT86 & 14 & 14,11 & 14 & 28 & 23 & 10 & 10 & 13 & 1 & $21 / 19$ & 13 & 1 \\
\hline HPT87 & 15 & 16,16 & 14 & 27 & 25 & 11 & 11 & 11 & 1 & $22 / 21$ & 13 & 1 \\
\hline HPT88 & 13 & $17.3,17$ & 14 & 28 & 25 & 11 & 11 & 11 & 1 & $22 / 18$ & 13 & 1 \\
\hline HPT89 & 16 & 14,14 & 13 & 26 & 24 & 11 & 11 & 13 & 1 & $20 / 20$ & 13 & 1 \\
\hline HРT90 & 16 & 13,11 & 14 & 28 & 26 & 11 & 11 & 11 & 1 & $21 / 19$ & 13 & 1 \\
\hline HРT91 & 15 & 14,14 & 13 & 27 & 23 & 11 & 11 & 15 & 1 & $19 / 18$ & 13 & 1 \\
\hline HРT92 & 13 & $17.3,12$ & 14 & 28 & 25 & 11 & 11 & 9 & 1 & $22 / 19$ & 16 & 1 \\
\hline HРT93 & 15 & 15,12 & 14 & 28 & 23 & 11 & 11 & 15 & 1 & $19 / 17$ & 13 & 1 \\
\hline HPT94 & 14 & 15,12 & 14 & 27 & 25 & 12 & 12 & 11 & 1 & $23 / 19$ & 13 & 1 \\
\hline
\end{tabular}




\begin{tabular}{|c|c|c|c|c|c|c|c|c|c|c|c|c|}
\hline HPT95 & 14 & 12,11 & 15 & 28 & 25 & 11 & 12 & 11 & 1 & $20 / 19$ & 13 & 1 \\
\hline НРT96 & 15 & 14,12 & 14 & 28 & 25 & 12 & 12 & 9 & 1 & $23 / 19$ & 13 & 1 \\
\hline НРT97 & 16 & 14,13 & 14 & 28 & 24 & 11 & 12 & 13 & 1 & $20 / 19$ & 15 & 1 \\
\hline HРT98 & 17 & 15,13 & 15 & 30 & 25 & 11 & 11 & 13 & 1 & $20 / 20$ & 13 & 1 \\
\hline HPT99 & 14 & 17,16 & 16 & 32 & 26 & 11 & 10 & 13 & 1 & $21 / 18$ & 13 & 1 \\
\hline HPT100 & 15 & 14,13 & 15 & 30 & 27 & 10 & 10 & 12 & 1 & $23 / 20$ & 16 & 1 \\
\hline HPT101 & 16 & 16,15 & 16 & 30 & 25 & 11 & 13 & 13 & 1 & $23 / 23$ & 13 & 1 \\
\hline HPT102 & 16 & 16,13 & 14 & 29 & 23 & 11 & 11 & 15 & 1 & $20 / 19$ & 13 & 1 \\
\hline HPT103 & 16 & 15,14 & 15 & 28 & 24 & 11 & 12 & 10 & 1 & $21 / 19$ & 13 & 1 \\
\hline HPT104 & 15 & 14,10 & 14 & 28 & 27 & 12 & 13 & 11 & 1 & $23 / 19$ & 13 & 1 \\
\hline HPT105 & 15 & 17,13 & 15 & 29 & 25 & 11 & 12 & 10 & 1 & $22 / 19$ & 16 & 1 \\
\hline HPT106 & 14 & 17,15 & 14 & 29 & 23 & 10 & 11 & 11 & 1 & $20 / 18$ & 13 & 1 \\
\hline HPT107 & 15 & 13,12 & 15 & 29 & 26 & 11 & 13 & 10 & 1 & $23 / 19$ & 13 & 1 \\
\hline HPT108 & 16 & 15,13 & 14 & 28 & 22 & 11 & 13 & 15 & 1 & $20 / 19$ & 13 & 1 \\
\hline HPT109 & 17 & $17.3,17$ & 16 & 31 & 23 & 11 & 12 & 15 & 1 & $21 / 19$ & 13 & 1 \\
\hline HPT110 & 17 & 14,13 & 14 & 28 & 23 & 12 & 11 & 14 & 1 & $20 / 19$ & 13 & 1 \\
\hline HPT111 & 15 & $17.3,14$ & 16 & 30 & 25 & 11 & 12 & 12 & 1 & $22 / 19$ & 14 & 1 \\
\hline HPT112 & 15 & 16,15 & 14 & 28 & 24 & 11 & 12 & 13 & 1 & $21 / 19$ & 15 & 1 \\
\hline HPT113 & 16 & $17.3,13$ & 15 & 29 & 24 & 11 & 13 & 12 & 1 & $22 / 19$ & 16 & 1 \\
\hline HPT114 & 16 & 17,11 & 15 & 29 & 25 & 11 & 11 & 12 & 1 & $22 / 19$ & 16 & 1 \\
\hline HPT115 & 15 & 16,15 & 16 & 30 & 26 & 10 & 12 & 14 & 1 & $22 / 19$ & 13 & 1 \\
\hline HPT116 & 18 & 19,13 & 16 & 29 & 25 & 11 & 11 & 13 & 1 & $21 / 19$ & 13 & 1 \\
\hline HPT117 & 15 & 15,14 & 15 & 28 & 25 & 12 & 12 & 12 & 1 & $22 / 19$ & 13 & 1 \\
\hline HPT118 & 17 & 14,10 & 16 & 31 & 27 & 11 & 11 & 13 & 1 & $23 / 19$ & 13 & 1 \\
\hline HPT119 & 16 & 14,10 & 15 & 29 & 25 & 13 & 12 & 13 & 1 & $22 / 19$ & 13 & 1 \\
\hline HPT 120 & 17 & 13,13 & 14 & 29 & 25 & 10 & 11 & 13 & 1 & $23 / 22$ & 14 & 1 \\
\hline HPT121 & 15 & 15,13 & 15 & 29 & 24 & 11 & 11 & 12 & 1 & $22 / 19$ & 16 & 1 \\
\hline HPT122 & 16 & 16,13 & 14 & 28 & 23 & 10 & 11 & 14 & 1 & $20 / 20$ & 13 & 1 \\
\hline HPT123 & 15 & 14,10 & 16 & 30 & 24 & 12 & 12 & 11 & 1 & $22 / 19$ & 13 & 1 \\
\hline HPT124 & 15 & 13,12 & 14 & 28 & 24 & 11 & 13 & 13 & 1 & $19 / 14$ & 13 & 1 \\
\hline HPT125 & 14 & 16,10 & 13 & 29 & 23 & 11 & 11 & 13 & 1 & $22 / 19$ & 13 & 1 \\
\hline HPT126 & 16 & $16.3,13$ & 14 & 29 & 23 & 11 & 11 & 13 & 1 & $22 / 19$ & 16 & 1 \\
\hline HPT127 & 15 & $17.3,13$ & 14 & 28 & 24 & 11 & 11 & 12 & 1 & $22 / 19$ & 16 & 1 \\
\hline HPT128 & 15 & 15,13 & 14 & 29 & 24 & 12 & 11 & 12 & 1 & $22 / 22$ & 18 & 1 \\
\hline HPT129 & 14 & $16.3,14$ & 14 & 29 & 23 & 13 & 13 & 13 & 1 & $19 / 19$ & 13 & 1 \\
\hline HPT130 & 15 & $17.3,17.3$ & 14 & 29 & 24 & 12 & 11 & 12 & 1 & $22 / 21$ & 15 & 1 \\
\hline HPT131 & 16 & $16.3,11$ & 14 & 28 & 24 & 12 & 11 & 12 & 1 & $22 / 19$ & 15 & 1 \\
\hline HPT132 & 16 & 13,10 & 13 & 29 & 24 & 11 & 11 & 14 & 1 & $20 / 20$ & 13 & 1 \\
\hline HPT133 & 15 & 16,10 & 13 & 28 & 23 & 11 & 9 & 13 & 1 & $20 / 20$ & 15 & 1 \\
\hline HPT134 & 15 & $17.3,15$ & 15 & 32 & 24 & 11 & 12 & 13 & 1 & $20 / 20$ & 13 & 1 \\
\hline HPT135 & 16 & 15,10 & 14 & 29 & 26 & 10 & 12 & 14 & 1 & $23 / 19$ & 14 & 1 \\
\hline HPT136 & 16 & 15,13 & 14 & 28 & 24 & 11 & 13 & 15 & 1 & $20 / 19$ & 14 & 1 \\
\hline HPT137 & 16 & 16,15 & 14 & 28 & 23 & 11 & 11 & 12 & 1 & $23 / 20$ & 13 & 1 \\
\hline HPT138 & 16 & 13,12 & 14 & 27 & 24 & 10 & 11 & 12 & 1 & $19 / 18$ & 17 & 1 \\
\hline HPT139 & 16 & 17,13 & 13 & 28 & 25 & 11 & 11 & 12 & 1 & $20 / 19$ & 13 & 1 \\
\hline HPT140 & 15 & 14,11 & 16 & 29 & 26 & 12 & 12 & 12 & 1 & $23 / 22$ & 13 & 1 \\
\hline HPT141 & 16 & 14,13 & 15 & 30 & 23 & 11 & 11 & 14 & 1 & $20 / 19$ & 13 & 1 \\
\hline HPT142 & 15 & 17,16 & 16 & 30 & 24 & 11 & 11 & 13 & 1 & $22 / 21$ & 13 & 1 \\
\hline HPT 143 & 16 & 16,13 & 16 & 31 & 24 & 10 & 11 & 13 & 1 & $22 / 19$ & 13 & 1 \\
\hline HPT144 & 16 & 13,12 & 16 & 31 & 24 & 11 & 10 & 13 & 1 & $20 / 19$ & 13 & 1 \\
\hline HPT145 & 16 & 17,16 & 16 & 32 & 22 & 11 & 11 & 12 & 1 & $21 / 20$ & 13 & 1 \\
\hline HPT146 & 16 & 14,13 & 15 & 30 & 25 & 12 & 11 & 13 & 1 & $22 / 21$ & 14 & 1 \\
\hline HPT147 & 16 & 14,13 & 13 & 29 & 23 & 11 & 11 & 14 & 1 & $20 / 19$ & 13 & 1 \\
\hline HPT148 & 16 & 11,11 & 15 & 32 & 24 & 12 & 12 & 13 & 1 & $22 / 18$ & 13 & 1 \\
\hline HPT149 & 15 & 14,11 & 14 & 30 & 25 & 11 & 12 & 12 & 1 & $22 / 18$ & 13 & 1 \\
\hline HPT150 & 15 & $17.3,13$ & 14 & 30 & 24 & 11 & 11 & 12 & 1 & $22 / 21$ & 17 & 1 \\
\hline HPT151 & 15 & 14,13 & 13 & 30 & 24 & 12 & 12 & 13 & 1 & $18 / 17$ & 16 & 1 \\
\hline HPT152 & 16 & 14,12 & 13 & 32 & 25 & 11 & 11 & 12 & 1 & $19 / 18$ & 15 & 1 \\
\hline HPT153 & 15 & 14,11 & 15 & 31 & 25 & 12 & 12 & 12 & 1 & $18 / 18$ & 15 & 1 \\
\hline HPT154 & 15 & 15,10 & 11 & 29 & 25 & 11 & 11 & 13 & 1 & $20 / 18$ & 15 & 1 \\
\hline HPT155 & 16 & 16,15 & 15 & 32 & 23 & 11 & 11 & 14 & 1 & $20 / 19$ & 13 & 1 \\
\hline HPT156 & 16 & 16,13 & 14 & 32 & 25 & 11 & 11 & 12 & 1 & $21 / 18$ & 15 & 1 \\
\hline HPT157 & 16 & 14,12 & 13 & 30 & 24 & 11 & 12 & 12 & 1 & $21 / 18$ & 18 & 1 \\
\hline HPT 158 & 14 & 15,14 & 13 & 30 & 25 & 11 & 11 & 13 & 1 & $21 / 19$ & 13 & 1 \\
\hline HPT 159 & 16 & 13,11 & 14 & 29 & 24 & 11 & 11 & 12 & 1 & $20 / 19$ & 15 & 1 \\
\hline HPT160 & 15 & 16,12 & 13 & 30 & 23 & 11 & 11 & 12 & 1 & $21 / 20$ & 14 & 1 \\
\hline HPT161 & 14 & 15,13 & 13 & 30 & 24 & 11 & 11 & 14 & 1 & $22 / 19$ & 14 & 1 \\
\hline HPT 162 & 14 & 14,14 & 13 & 30 & 25 & 10 & 11 & 13 & 1 & $22 / 19$ & 13 & 1 \\
\hline HPT163 & 14 & 15,13 & 13 & 28 & 23 & 11 & 15 & 13 & 1 & $19 / 19$ & 13 & 1 \\
\hline
\end{tabular}




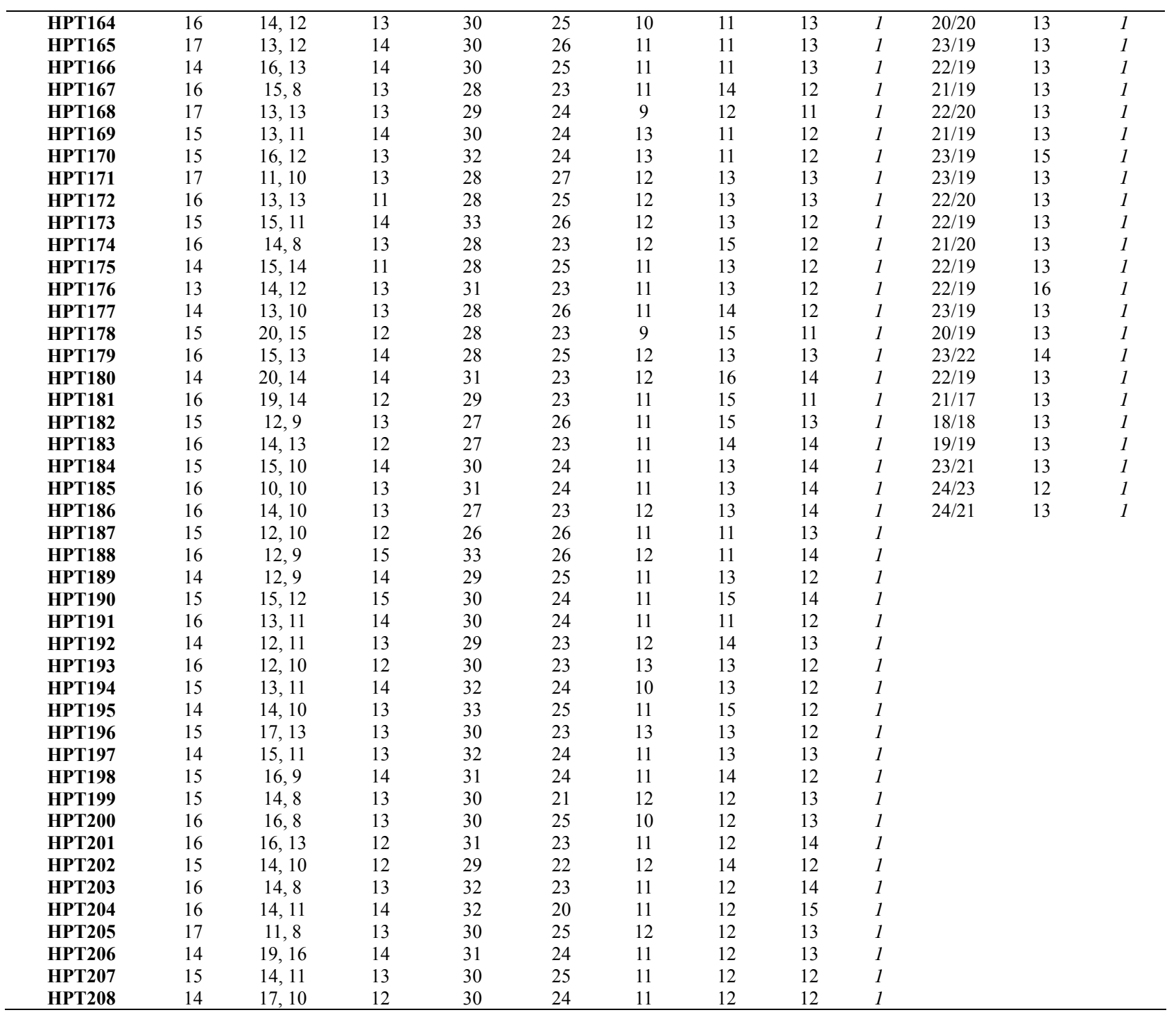

*Haplotype containing 10 Y-STR loci; **Haplotype containing 8 Y-STR loci; ***Number of individuals bearing each haplotype.

Table 3. Comparative presentation of haplotype numbers, gene diversities, average gene diversities and $P_{d}$ combined values belonging to three haplotypes surveyed in this study.

\begin{tabular}{ccccc}
\hline Haplotypes & $\mathbf{n}$ & Gene diversity & $\begin{array}{c}\text { Average gene } \\
\text { diversity per locus }\end{array}$ & PDc* $^{*}$ \\
\hline Ht10 & 186 & $1.0000 \pm 0.0006$ & 0.7476 & 0.9999989 \\
MinHt & 208 & $1.0000 \pm 0.0005$ & 0.7518 & 0.9999869 \\
$a H t$ & 186 & $1.0000 \pm 0.0006$ & 0.7834 & 0.9999936 \\
\hline
\end{tabular}

*Combined Power of Discrimination.

$(\mathrm{n}=166)$ [26], Czech $(\mathrm{n}=50)$ [27], German $(\mathrm{n}=166)$, Indian $(\mathrm{n}=108)$, Mozambican $(\mathrm{n}=112)$ [12], Japanese $(\mathrm{n}=161)$ [28], Turkish (Antalya) $(\mathrm{n}=210)$ [29], and Turkish $(\mathrm{n}=280)$ [30]. Haplotypic comparisons have highlighted that no significant difference has been observed with Czech population $(p>0.05)$ while comparisons with all other populations have produced statistically significant differences from Turkish population in this study ( $p<0.01)$, as shown in Table 4.

Our results suggest that an alternative haplotype $(a H t)$, which differs slightly from minHt in respect of its Y-STR loci contents, maybe alternative for minHt in Turkish population. $a H t$ has included the selected 8 Y-STR loci: DYS19, DYS385a/b, DYS389I, DYS389II, DYS390, DYS392, DYS393, and YCAIIa/b. The only difference between the minHt and the proposed $a H t$ is the inclusion of YCAIIa/b locus in place of DYS391 locus in aHt due to its higher gene diversity value of 0.8087 as compared with 0.6101 of DYS391 locus (Table 1). We have found 186 unique haplotype in $a H t$ (data not shown). The $a H t$ has reflected a better $P_{d}$ combined value when compared with minHt (0.9999936 vs 0.9999869$)$ and, has higher average gene diversity per locus (0.7834 vs 0.7518$)$ (Table 3). The data has exhibited that $a H t$ has a higher discriminatory potential than that of $\operatorname{minHt}$. 
Table 4. Exact test $\mathrm{P}$ values of populational genetic differentiation measures based on minHt haplotype frequencies between all pairs involving samples of eight populations and Turkish population in this study.

\begin{tabular}{|c|c|c|c|c|c|c|c|c|c|}
\hline Population & $\begin{array}{c}\text { Turkish } \\
\text { (This study) }\end{array}$ & Croatian & Czech & German & Indian & Mozambican & Japanese & Turkish & $\begin{array}{c}\text { Turkish } \\
\text { (Antalya) }\end{array}$ \\
\hline Turkish (This study) & - & & & & & & & & \\
\hline $\operatorname{Czech}^{27}$ & $\begin{array}{l}0.32474 \\
\pm 0.0283\end{array}$ & - & & & & & & & \\
\hline Mozambican $^{12}$ & 0.0000 & $\begin{array}{l}0.00103 \\
\pm 0.0010\end{array}$ & - & & & & & & \\
\hline Croatian $^{26}$ & 0.0000 & 0.0000 & 0.0000 & - & & & & & \\
\hline Indian $^{12}$ & 0.0000 & 0.0000 & 0.0000 & 0.0000 & - & & & & \\
\hline Japanese $^{28}$ & $\begin{array}{l}0.00205 \\
\pm 0.0017\end{array}$ & $\begin{array}{l}0.01176 \\
\pm 0.0026\end{array}$ & 0.0000 & 0.0000 & 0.0000 & - & & & \\
\hline German $^{12}$ & 0.0000 & 0.0000 & 0.0000 & 0.0000 & 0.0000 & 0.0000 & - & & \\
\hline Turkish $^{30}$ & 0.0000 & 0.0000 & 0.0000 & 0.0000 & 0.0000 & 0.0000 & 0.0000 & - & \\
\hline $\begin{array}{c}\text { Turkish } \\
\text { (Antalya) }^{29}\end{array}$ & 0.0000 & 0.0000 & 0.0000 & 0.0000 & 0.0000 & 0.0000 & 0.0000 & 0.0000 & - \\
\hline
\end{tabular}

In conclusion this study results have yielded sufficient evidence that aHt can reliably be proposed as an alternative to minHt in paternity testing and forensic medicine applications for Turkish population. Our data have also provided additional information to the framework of variation involving $10 \mathrm{Y}$-STR loci as well as a further contribution to the Y-STR database for Turkish population.

\section{ACKNOWLEDGEMENTS}

This study was supported by Ankara University Scientific Research Projects with the project \# 2005-08-09-012 HPD and approved by the Ankara University, Research Ethics Committee of Medical Faculty with the approval \# 57-1393.

This study was performed in Ankara University, Medical Faculty, Medical Biology Department.

\section{REFERENCES}

[1] de Knijff, P. (2000) Messages through bottlenecks: On the combined use of slow and fast evolving polymorphic markers on the human Y chromosome. The American Journal of Human Genetics, 67, 1055-1061.

[2] Spurdle, A.B., Woodfield, D.G., Hammer, M.F. and Jenkins, T. (1994) The genetic affinity of Polynesians: Evidence from Y chromosome polymorphisms. Annals of Human Genetics, 58, 251-263. doi:10.1111/j.1469-1809.1994.tb01889.x

[3] Dupuy, B.M., Stenersen, M., Egeland, T. and Ola1sen, B. (2004) Y-chromosomal microsatellite mutation rates: Differences in mutation rate between and within loci. Hum Mut, 23, 117-124. doi:10.1002/humu.10294

[4] Gill, P., Brenner, C., Brinkmann, B., Budowle, B., Carracedo, A., Jobling, M.A., de Knijff, P., Kayser, M., Krawczak, M., Mayr, W.R., Morling, N., Olaisen, B., Pascali, V., Prinz, M., Roewer, L., Schneider, P.M., Sajantila, A. and Tyler-Smith, C. (2001) DNA Commission of the International Society of Forensic Genetics: Recommendations on forensic analysis using Y-chromosome STRs. Forensic Science International, 124, 5-10. doi:10.1016/S0379-0738(01)00498-4

[5] Betz, A., Bäbler, G., Dietl, G., Syeil, X., Weyermann, G. and Pflug, W. (2001) DYS STR analysis with epithelial cells in a rape case. Forensic Science International, 118, 126-130. doi:10.1016/S0379-0738(00)00482-5

[6] Corach, D., Figueira, R.L., Marino, M., Penacino, G. and Sala, A. (2001) Routine Y-STR typing in forensic casework. Forensic Science International, 118, 131-135. doi:10.1016/S0379-0738(00)00483-7

[7] Dekairelle, A.F. and Hoste, B. (2001) Application of a Y-STR-pentaplex PCR (DYS19, DYS389I and II, DYS390 and DYS393) to sexual assault cases. Forensic Science International, 118, 122-125. doi:10.1016/S0379-0738(00)00481-3

[8] Gill, P., Werrett, D.J., Budowle, B. and Guerrieri, R. (2004) An assessment of whether SNPs will replace STRs in national DNA databases-joint considerations of the DNA working group of the European Network of Forensic Science Institutes (ENFSI) and the Scientific Working Group on DNA Analysis Methods (SWGDAM). Science \& Justice, 44, 51-3. doi:10.1016/S1355-0306(04)71685-8

[9] Honda, K., Tun, Z., Young, D. and Terao, T. (2001) Examination of Y-STR mutations in sex chromosomal abnormality in forensic case. Forensic Science International, 118, 136-140. doi:10.1016/S0379-0738(00)00484-9

[10] Jobling, M.A., Pandya, A. and Tyler-Smith, C. (1997) The $\mathrm{Y}$ chromosome in forensic analysis and paternity testing. International Journal of Legal Medicine, 110, 118-124. doi:10.1007/s004140050050

[11] Kayser, M. and Sajantila, A. (2001) Mutations at Y-STR loci: Implications for paternity testing and forensic analysis. Forensic Science International, 118, 116-121. doi:10.1016/S0379-0738(00)00480-1

[12] Alaves, C., Gusmao, L., Barbosa, J. and Amorim, A. (2003) Evaluating the informative power of Y-STRs: A comparative study using European and new African haplotype data. Forensic Science International, 134, 126133. doi:10.1016/S0379-0738(03)00127-0

[13] Gusmao, L., Sanchez-Diz, P., Alves, C., Beleza, S., Lopes, A., Carracedo, A. and Amorim, A. (2003) Grouping of Y-STR haplotypes discloses European geografic clines. Forensic Science International, 134, 172-179. doi:10.1016/S0379-0738(03)00160-9

[14] Koyama, H., Iwasa, M., Maeno, Y., Tsuchimochi, T., 
Isobe, I., Yoshimi, S.N., Jun, M.O., Matsumoto, T., Horii, T. and Nagao, M. (2001) Y-chromosomal STR haplotype in the Japanese population. Forensic Science International, 124, 221-223. doi:10.1016/S0379-0738(01)00572-2

[15] Nakagome, Y., Young, S.R., Akane, A., Numabe, H., Jin, D.K., Yamori, Y., Seki, S., Tamura, T., Nagafuchi, S., Shiono, H. and Nakahori, Y. (1992) A Y-associated alele may be characteristic of cetain ethnic groups in Asia. Annals of Human Genetics, 56, 311-314. doi:10.1111/j.1469-1809.1992.tb01158.x

[16] Rodig, H., Grum, M. and Grimmecke, H.-D. (2007) Population study and evaluation of 20 Y-chromosome STR loci in Germans. International Journal of Legal Medicine, 121, 24-27. doi:10.1007/s00414-005-0075-5

[17] Rustamov, A., Gümüş, G., Karabulut, H.G., Elhan, A.H., Kadıkıran, A. and Bökesoy, I. (2004) Y-STR polymorphism in Central Anatolian region of Turkey. Forensic Science International, 139, 227-230. doi:10.1016/j.forsciint.2003.11.009

[18] Yan, J., Tang, H., Liu, Y., Jing, Y., Jiao, Z., Zhang, Q., Gao, J., Shang, L., Guo, H. and Yu, J. (2007) Genetic polymorphisms of 17 Y-STRs haplotypes in Chinese Han population residing in Shandong province of China. Legal Medicine, 9, 196-202. doi:10.1016/j.legalmed.2006.11.016

[19] Park, M.J., Lee, H.Y, Chung, U., Kang, S.-C. and Shin, K.-J. (2007) Y-STR analysis of degraded DNA using reduced-size amplicons. International Journal of Legal Medicine, 121, 152-157. doi:10.1007/s00414-006-0133-7

[20] Willuweit, S. and Roewer, L. (2007) Y chromosome haplotype reference database (YHRD): Update. Forensic Science International, 1, 83-87. doi:10.1016/j.fsigen.2007.01.017

[21] YHRD-Y Chromosome Haplotype Reference Database. (2011). http://www.yhrd.org
[22] Sambrook, J. and Russell, D. (2001) Molecular Cloning: A Laboratory Manual. 3rd Edition, Cold Spring Harbor Laboratory, Cold Spring Harbor, New York, 543-554.

[23] Santos, F.R., Pena, D.J. and Epplen, J.T. (1993) Genetic and population study of a Y-linked tetranucleotide repeat DNA polymorphism with a simple non-isotopic technique. Human Genetics, 90, 655-656. doi:10.1007/BF00202486

[24] Nei, M. (1996) Phylogenetic analysis in molecular evolutionary genetics. Annual Review of Genetics, 30, 371-403 doi:10.1146/annurev.genet.30.1.371

[25] Kimberly, A.H. (2001) Statistical analyses of STR data profiles in DNA.

http://promega.de/profiles/103/Profile-sinDNA_103_14. pdf.

[26] Ljubkovic, J., Stripisic, A., Sutlovic, D., Definis-Gojanovic, M., Bucan, K. and Andelinovic, S. (2008) Y-chromosomal short tandem repeat haplotypes in southern croatian male population defined by 17 loci. Croatian Medical Journal, 49, 201-206. doi:10.3325/cmj.2008.2.201

[27] Ehler, E., Marvan, R. and Vanek, D. (2010) Evaluation of $14 \mathrm{Y}$ chromosomal short tandem repeat haplotype with focus on DYS449, DYS456, and DYS458: Czech population sample. Croatian Medical Journal, 51, 54-60. doi:10.3325/cmj.2010.51.54

[28] Hara, M., Kido, A., Takada, A., Adachi, N. and Saito, K. (2007) Genetic data for 16 Y-chromosomal STR loci in Japanese. Legal Medicine, 9, 161-170. doi:10.1016/j.legalmed.2006.11.002

[29] Demirçin, S. and Timur, S. (2010) Haplotype analysis of 8 Y-STR loci in the Antalya population. Romanian Journal of Legal Medicine, 18, 51-58.

[30] Henke, J., Henke, L., Chatthopadhyay, P., Kayser, M., Dulmer, M., Cleef, S., Poche, H. and Felske, Z.H. (2001) Application of Y-chromosomal STR haplotypes to forensic genetics. Croatian Medical Journal, 42, 292297. 